ELIENER de SOUZA FAZIO'

ROSEI MIEKO YAMAMOTO NOMURA ${ }^{2}$

Maria Carolina Gonçalves Dias ${ }^{3}$

MARCELO ZUGAiB ${ }^{4}$

Artigo original

Palavras-chave

Consumo de alimentos Comportamento alimentar Índice de massa corporal Avaliação nutricional

Ganho de peso

Gravidez

Keywords

Food consumption

Feeding behavior

Body mass index Nutritional assessment

Weight gain

Pregnancy

\title{
Consumo dietético de gestantes e ganho ponderal materno após aconselhamento nutricional
}

\author{
Dietary intake of pregnant women and maternal weight gain after \\ nutritional counseling
}

Resumo

OBJETIVOS: conhecer o consumo dietético de gestantes avaliando a ingestão de macronutrientes e micronutrientes, e verificar o ganho ponderal materno na gravidez. MÉTODOS: estudo retrospectivo do período de junho de 2002 a junho de 2008 com gestantes que receberam orientação nutricional durante pré-natal em hospital universitário, agrupadas de acordo com o estado nutricional antropométrico classificado pelo índice de massa corpórea (IMC) pré-gestacional. $\bigcirc$ consumo dietético foi analisado pelas informações de entrevista de frequência alimentar realizada na primeira avaliação da gestante no serviço de nutrição, para obter os dados do hábito alimentar, calculando-se a ingestão de macronutrientes e micronutrientes. As gestantes receberam aconselhamento nutricional, e foi analisado o ganho ponderal materno na gravidez. RESULTADOS: do total de 187 gestantes que receberam orientação nutricional, 23 (12,2\%) eram de baixo peso, $84(45,0 \%)$, eutróficas, $37(19,8 \%)$ com sobrepeso, e 43 (23,0\%), obesas. As gestantes de baixo peso apresentaram menor consumo de lípides quando comparadas ao grupo com eutrofia (101,4 versus 137,3 g; p=0,043). A média do consumo de ferro foi maior nas gestantes eutróficas $(14,6 \mathrm{mg} / \mathrm{d})$ quando comparadas às com sobrepeso $(12,2$ $\mathrm{mg} / \mathrm{d}$ ) ou obesidade (10,9 mg/d; $<<0,001)$. A média da ingestão de folatos foi maior nas gestantes eutróficas quando comparadas às obesas $(336,5$ versus $234,5 \mathrm{\mu g} / \mathrm{d} ; \mathrm{p}=0,002)$. $\bigcirc$ ganho de peso excessivo, acima do recomendado, foi significativamente mais frequente $(p=0,009)$ nas gestantes com sobrepeso $(56,7 \%)$ e obesidade $(39,5 \%)$ quando comparadas às com baixo peso $(17,4 \%)$ e eutrofia $(31,0 \%)$. CONCLUSÕES: o ganho ponderal materno acima do recomendado associou-se ao sobrepeso e à obesidade. $\bigcirc$ consumo dietético de gestantes difere conforme o estado nutricional antropométrico materno, com menor ingestão diária de ferro nas gestantes com sobrepeso e obesidade, e menor ingestão de folatos nas obesas, o que reforça a importância da suplementação vitamínica pré-natal.

\section{Abstract}

PURPOSE: to determine the dietary consumption of pregnant women, by assessing the intake of macronutrients and micronutrients, and to verify the maternal weight gain during pregnancy. METHODS: a retrospective study conducted from June 2002 to June 2008 with pregnant women who received nutritional counseling during prenatal care at a university hospital, grouped according to anthropometric nutritional status classified by pregestational body mass index (BMI). The dietary intake was analyzed according to the information obtained in food frequency interviews, performed at the first evaluation of pregnant women in the service of nutrition to obtain data about eating habits, and the intake of macronutrients and micronutrients was calculated. The pregnant women received nutritional counseling, and the maternal weight gain was investigated. RESULTS: a total of 187 pregnant women who received nutritional counseling were analyzed. Twenty-three (12.2\%) were underweight, 84 (45.0\%) normal weight, 37 (19.8\%) overweight, and 43 (23.0\%) obese. The underweight pregnant women had lower consumption of lipids when compared to the normal weight group 1101.4 versus 137.3 g; $p=0.043)$. The average iron intake was higher in normal weight pregnant women $(14.6 \mathrm{mg} / \mathrm{d})$ compared to the overweight (12.2 mg/d) or obese $(10.9 \mathrm{mg} / \mathrm{d} ; \mathrm{p}<0.001)$ groups. The average intake of folate was higher in normal weight pregnant women compared to obese ones $(336.5 \mathrm{\mu g} / \mathrm{d}$ versus $234.5 \mathrm{\mu g} / \mathrm{d} ; \mathrm{p}=0.002)$. Excessive maternal

Correspondência:

Roseli Mieko Yamamoto Nomuro Departamento de Obstetríia e Ginecologia, Faculdade de Medicina da Universidade de São Paulo - USP -

São Paulo (SP), Brasil

Av. Dr. Enéas de Carvalho Aguiar $255-10^{\circ}$ andar - sala 10.037 (EPP: 05403-000, São Paulo (SP), Brasil Tel. (11) 3069-6209 / Fax (11) 3069-8183 E-mail: roseli.nomur@@hotmail.com

Recebido

$17 / 08 / 2010$

Aceito com modificacọoes

$22 / 02 / 2011$
Disciplina de Obstetrícia do Departamento de Obstetrícia e Ginecologia da Faculdade de Medicina da Universidade de São Paulo - USP - São Paulo (SP), Brasil; Divisão de Nutrição e Dietética do Hospital das Clínicas da Faculdade de Medicina da Universidade de São Paulo São Paulo - USP - São Paulo (SP), Brasil.

' Nutricionista, Pós-graduanda do Departamento de Obstetrícia e Ginecologia da Faculdade de Medicina da Universidade de São Paulo - USP - São Paulo (SP), Brasil.

2 Professor do Departamento de Obstetrícia e Ginecologia da Faculdade de Medicina da Universidade de São Paulo - USP - São Paulo (SP), Brasil.

Nutricionista, Mestre em Ciências, Divisão de Nutrição e Dietética do Hospital das Clínicas da Faculdade de Medicina da Universidade de São Paulo - USP - São Paulo (SP), Brasil.

4 Professor Titular do Departamento de Obstetrícia e Ginecologia da Faculdade de Medicina da Universidade de São Paulo - USP - São Paulo (SP), Brasil. 
weight gain was significantly ( $\mathrm{p}=0.009)$ more frequent in overweight $(56.7 \%)$ and obese $(39.5 \%)$ pregnant women compared to underweight $(17.4 \%)$ and normal weight (31.0\%) women. CONCLUSIONS: The maternal weight gain above recommended levels was associated with overweight and obesity. The dietary intake of pregnant women differs according to maternal anthropometric nutritional status, with a lower daily intake of iron in overweight and obese women and a lower intake of folate in obese ones, a fact that reinforces the importance of prenatal vitamin supplementation.

\section{Introdução}

As alterações nutricionais e metabólicas que ocorrem durante a gestação visam proporcionar ambiente favorável para o desenvolvimento normal do concepto. Essas modificações fisiológicas promovem alterações no organismo materno preparando-o para a maternidade. O conhecimento da fisiologia do estado gravídico é fundamental para a melhor condução do atendimento pré-natal.

O período gestacional é heterogêneo em seus aspectos fisiológicos, metabólicos e nutricionais. No primeiro trimestre, a saúde do embrião vai depender da condição nutricional pré-gestacional da mãe, não somente quanto às suas reservas de energia, mas também quanto às de vitaminas, minerais e oligoelementos. O segundo e terceiro trimestres integram outra etapa para a gestante, em que as condições ambientais vão exercer influência direta no estado nutricional do feto ${ }^{1-3}$.

As gestantes são suscetíveis à inadequação nutricional, pelo aumento da demanda de energia, macro e micronutrientes, que ocorrem durante a gravidez, a fim de se garantir a saúde materno-fetal ${ }^{4,5}$. A qualidade da alimentação e o estado nutricional antropométrico da mulher, antes e durante a gravidez, afetam o crescimento e o desenvolvimento fetal, bem como a evolução da gestação.

Tem sido observado na nossa população, pela análise do consumo dietético, desequilíbrio na ingestão de nutrientes ${ }^{6,7}$. Durante a gestação, esse desequilíbrio pode implicar no comprometimento do crescimento e desenvolvimento do concepto, bem como no ganho de peso na gravidez, tornando de grande interesse o estudo da evolução ponderal da gestante e dos fatores relacionados ${ }^{8-12}$.

Esta pesquisa teve como objetivo conhecer o consumo dietético de gestantes atendidas em hospital universitário, avaliando a ingestão de macronutrientes e micronutrientes, e verificar o ganho ponderal materno na gravidez de acordo com o estado nutricional antropométrico.

\section{Métodos}

Este trabalho foi realizado em hospital universitário e abrangeu o período compreendido entre junho de 2002 e junho de 2008. A pesquisa teve seu projeto apresentado e aprovado pela Comissão de Ética em Pesquisa da instituição, sob número 0917/08.

Foi realizada pesquisa retrospectiva das informações de gestantes encaminhadas para a equipe de nutrição e dietética de acordo com a rotina assistencial estabelecida na instituição. As gestantes de baixo peso, sobrepeso ou obesas foram encaminhadas pelos ambulatórios de pré-natal de baixo, médio e alto risco para orientação da qualidade da alimentação, bem como acompanhamento do ganho de peso materno; as gestantes eutróficas foram encaminhadas por sinais ou sintomas relacionados à alimentação, informados pela paciente ao médico durante a consulta de pré-natal ou por livre demanda.

Foram utilizados os seguintes critérios de inclusão: gestantes com idade maior ou igual a 18 anos completos; ausência de diagnóstico prévio de doença materna; gestação única; ausência de malformação ou anomalia fetal; orientação iniciada pela equipe de nutrição até a $30^{a}$ semana de gestação; pré-natal e parto realizados na instituição.

Os dados foram coletados retrospectivamente por meio de consulta às fichas de evolução dietoterápica, padronizadas pela equipe de nutrição e dietética, e pela consulta de prontuários das pacientes. $\mathrm{Na}$ entrevista inicial realizada pela nutricionista, foram obtidas informações gerais da paciente, bem como peso e altura. $\mathrm{O}$ peso pré-gestacional foi informado pela paciente, ou, na ausência desta informação, foi utilizado o peso obtido na primeira consulta de pré-natal realizada até a $12^{\text {a }}$ semana de gestação. Para classificação do estado nutricional antropométrico pré-gestacional, foi utilizada a classificação proposta pelo Instituto de Medicina (IOM) da Academia Nacional de Ciências dos Estados Unidos ${ }^{13}$. Segundo esta classificação, considerou-se que mulheres com IMC pré-gestacional menor de $19,8 \mathrm{~kg} / \mathrm{m}^{2}$ eram de baixo peso; eutróficas, com IMC de 19,8 a $26,0 \mathrm{~kg} / \mathrm{m}^{2}$; sobrepeso, com IMC entre 26 a $29 \mathrm{~kg} / \mathrm{m}^{2}$; e obesas, com IMC acima de $29 \mathrm{~kg} / \mathrm{m}^{2}$. O ganho ponderal materno foi analisado segundo o estado nutricional antropométrico pré-gestacional pela classificação do IOM, que, para as gestantes de baixo peso, eutrofia, sobrepeso e obesidade, recomenda, respectivamente, ganho total de 12,5 a $18 \mathrm{~kg}$, de 11,5 a $16 \mathrm{~kg}$, de 7 a 11,5 kg, e de 7,0 a 9,1 kg.

Para avaliação do consumo dietético foram analisados os dados da entrevista de frequência alimentar, realizada na primeira avaliação da gestante no serviço de nutrição, antes de ser oferecida a orientação específica. Trata-se de questionário de frequência alimentar padronizado e adotado no Serviço de Nutrição e Dietética da instituição, que inclui questões sobre qualidade e quantidade dos alimentos, nos seguintes momentos: café da manhã (31 itens), lanche da manhã (6 itens), almoço (36 itens), 
lanche da tarde (6 itens), jantar (36 itens) e lanche noturno (6 itens). As informações dos alimentos e/ou preparações indicados em medidas caseiras pelas gestantes foram convertidos em gramas por meio da tabela para avaliação de consumo alimentar em medidas caseiras. Os dados sobre os alimentos consumidos (em gramas) na dieta foram analisados pela sua composição nutricional, utilizando-se tabelas de composição química de alimentos, que formam o software Nutwin (CIS EPM versão 2.5 n ${ }^{\circ} 2380$ ). Para os alimentos e/ou preparações que não constavam no banco de dados do Nutwin, foram considerados os valores da composição nutricional da Tabela Brasileira de Composição de Alimentos (TACO) e de tabelas de composição nutricional de rótulos dos alimentos.

As recomendações dos macronutrientes foram avaliadas de acordo com os valores determinados pela Organização Mundial da Saúde $(2003)^{14}$. Foram analisados os macronutrientes (carboidratos, proteínas e lípides) e os micronutrientes: cálcio, ferro, ácido fólico, vitaminas A e C, além das fibras. Estes valores serão analisados de acordo com as recomendações da Ingestão Dietética de Referência (DRI) ${ }^{15-17}$, para gestantes.

O ganho ponderal total foi calculado pela diferença entre o peso materno no dia do parto e o peso pré-gestacional. A idade gestacional foi calculada a partir da data da última menstruação (DUM), quando era compatível com a idade gestacional estimada pela ultrassonografia realizada, no máximo, até a vigésima semana de gestação. Nos casos em que não foi observada tal concordância, a idade gestacional foi calculada pelos dados da primeira ultrassonografia.

Os resultados foram analisados através do programa Statistica for Windows (versão 4.3, Statsoft ${ }^{\circledR}$, Inc., 1993). As variáveis foram analisadas descritivamente, calculandose frequências absolutas e relativas. Para as variáveis quantitativas, esta análise foi feita pela observação dos valores mínimos, máximos, cálculo de médias e desvios padrão. Para comparação entre proporções foi aplicado o teste do $\chi^{2}$, e, quando pertinente o Teste Exato de Fisher. Foi utilizado o teste ANOVA para a comparação das médias entre os grupos, nas variáveis com distribuição normal, e o Teste de Kruskal-Wallis para as variáveis de distribuição não normal. Para identificação da significância dos grupos estudados, foi aplicado o Teste de Tukey. Foi adotado como nível de significância o valor 0,05 (alfa =5\%). Com isso, níveis descritivos (p) inferiores a esse valor serão considerados significantes $(\mathrm{p}<0,05)$.

\section{Resultados}

Foram avaliadas 187 gestantes, com média de idade de 30,6 anos e desvio padrão de 6,2 anos. A média da idade gestacional na primeira avaliação nutricional foi de 21 semanas, com desvio padrão de 7 semanas. Setenta e cinco $(40,1 \%)$ gestantes eram nulíparas, $56(30,0 \%)$ eram primíparas, e $56(30,0 \%)$ apresentavam paridade superior a um. Do total de prontuários inicialmente selecionados, foram excluídos 64 casos de diagnóstico prévio de doença materna, 41 casos que foram referenciadas ao serviço de nutrição após a $30^{\mathrm{a}}$ semana, 27 casos de gestantes adolescentes, 18 casos que perderam o seguimento ou cujo parto foi realizado em outra instituição, cinco casos com diagnóstico de malformação fetal, e três casos de gestação gemelar, restando 187 casos para a presente investigação.

Das gestantes que receberam orientação nutricional incluídas, $23(12,2 \%)$ eram de baixo peso, $84(45,0 \%)$, eutróficas, 37 (19,8\%) com sobrepeso, e 43 (23,0\%), obesas. As gestantes classificadas como de baixo peso apresentaram média de idade inferior à observada nos

Tabela 1 - Características maternas, consumo energético e de macronutrientes de gestantes que receberam orientação dietética, de acordo com 0 estado nutricional antropométrico materno

\begin{tabular}{|c|c|c|c|c|c|c|c|c|c|}
\hline \multirow[t]{2}{*}{ Características } & \multicolumn{2}{|c|}{$\begin{array}{c}\text { Baixo peso } \\
(n=23)\end{array}$} & \multicolumn{2}{|c|}{$\begin{array}{l}\text { Eutrofia } \\
(n=84)\end{array}$} & \multicolumn{2}{|c|}{$\begin{array}{c}\text { Sobrepeso } \\
(n=37)\end{array}$} & \multicolumn{2}{|c|}{$\begin{array}{c}\text { Obesidade } \\
(n=43)\end{array}$} & \multirow[t]{2}{*}{ Valor $\mathrm{p}$} \\
\hline & Média & DP & Média & DP & Média & DP & Média & DP & \\
\hline Idade materna (anos) & 27,4 & $(5,7)$ & 30,4 & $(6,4)$ & 33,0 & $(5,3)$ & 30,6 & $(6,2)$ & $0,008^{\star}$ \\
\hline IMC pré-gestacional $\left(\mathrm{kg} / \mathrm{m}^{2}\right)$ & 18,2 & $(0,8)$ & 23,3 & $(1,6)$ & 27,4 & $(0,9)$ & 34,3 & $(4,6)$ & $<0,001$ \\
\hline IG (semanas) & 20,8 & $(5,5)$ & 23,5 & $(6,8)$ & 19,6 & $(7,5)$ & 19,3 & $(6,4)$ & $0,002^{\star \star}$ \\
\hline Carboidratos & 239,8 & $(56,4)$ & 288,1 & $(102,8)$ & 269,2 & $(88,4)$ & 269,2 & $(112,3)$ & 0,198 \\
\hline Proteínas & 69,1 & $(29,3)$ & 85,7 & $(23,6)$ & 84,0 & $(20,7)$ & 82,3 & $(36,4)$ & 0,082 \\
\hline Lípides & 101,4 & $(39,2)$ & 137,3 & $(48,7)$ & 132,2 & $(48,2)$ & 131,0 & $(69,7)$ & $0,043^{\star \star \star}$ \\
\hline \multicolumn{10}{|l|}{ Macronutrientes (\%) } \\
\hline Carboidratos & 59,0 & $(6,8)$ & 55,7 & $(7,1)$ & 54,9 & $(7,3)$ & 55,7 & $(9,0)$ & 0,211 \\
\hline
\end{tabular}

IMC: índice de massa corpórea; IG: idade gestacional; DP: desvio padrão.

*Baixo peso versus eutrofia, sobrepeso e obesidade; **Eutrofia versus baixo peso, sobrepeso e obesidade; ***Baixo peso versus eutrofia. 
Tabela 2 - Características do consumo de micronutrientes e fibras de gestantes que receberam orientação dietética, de acordo com 0 estado nutricional antropométrico materno

\begin{tabular}{|c|c|c|c|c|c|c|c|c|c|c|}
\hline \multirow[t]{2}{*}{ Micronutriente } & \multirow{2}{*}{$\begin{array}{l}\text { Necessidades } \\
\text { diárias }\end{array}$} & \multicolumn{2}{|c|}{$\begin{array}{c}\text { Baixo peso } \\
(n=23)\end{array}$} & \multicolumn{2}{|c|}{$\begin{array}{c}\text { Eutrofia } \\
(n=84)\end{array}$} & \multicolumn{2}{|c|}{$\begin{array}{c}\text { Sobrepeso } \\
(\mathrm{n}=37)\end{array}$} & \multicolumn{2}{|c|}{$\begin{array}{c}\text { Obesidade } \\
(n=43)\end{array}$} & \multirow[t]{2}{*}{ Valor $p$} \\
\hline & & Média & DP & Média & DP & Média & DP & Média & DP & \\
\hline Cálcio (mg) & $1000(\mathrm{Al})^{13}$ & 713,3 & 224,6 & 842,9 & 340,6 & 835,4 & 335,1 & 748,6 & 337,0 & 0,212 \\
\hline Ferro $(g)$ & $27(\mathrm{RDA})^{15}$ & 11,9 & 4,2 & 14,6 & 4,9 & 12,2 & 4,4 & 10,9 & 4,9 & $0,000 *$ \\
\hline Folato (ug) & $520(\text { EAR })^{13}$ & 254,9 & 122,2 & 336,5 & 165,2 & 274,2 & 148,1 & 234,5 & 149,2 & $0,002^{\star \star}$ \\
\hline Vitamina A (RE) & $550(\text { EAR })^{15}$ & 693,7 & 659,3 & 703,0 & 728,5 & 666,1 & 1015,0 & 519,8 & 440,1 & 0,598 \\
\hline Vitamina C (mg) & $70(\text { EAR) })^{16}$ & 162,1 & 128,8 & 139,7 & 123,8 & 246,9 & 650,9 & 137,3 & 132,6 & 0,327 \\
\hline Fibras (g) & $28(\mathrm{Al})^{17}$ & 17,0 & 5,3 & 22,8 & 8,9 & 20,7 & 9,2 & 20,2 & 10,4 & $0,042^{\star \star \star}$ \\
\hline
\end{tabular}

RE: retinol equivalente; $\mathrm{Al}$ : adequate intake; RDA: recommended dietary allowances; EAR: estimated average requirement.

${ }^{*} p=0,048$ - eutrofia versus sobrepeso; $p<0,001$ - eutrofia versus obesidade; ${ }^{*} p=0,002$ - eutrofia versus obesidade; ${ }^{*}{ }^{*} p=0,032$ - baixo peso versus eutrofia.

Tabela 3 - Classificação do ganho ponderal total de gestantes que receberam orientação dietética, de acordo com o estado nutricional antropométrico materno

\begin{tabular}{|c|c|c|c|c|c|c|c|c|c|}
\hline \multirow[t]{2}{*}{ Ganho ponderal total ${ }^{1}$} & \multicolumn{2}{|c|}{$\begin{array}{c}\text { Baixo peso } \\
(n=23)\end{array}$} & \multicolumn{2}{|c|}{$\begin{array}{l}\text { Eutrofia } \\
(n=84)\end{array}$} & \multicolumn{2}{|c|}{$\begin{array}{c}\text { Sobrepeso } \\
(n=37)\end{array}$} & \multicolumn{2}{|c|}{$\begin{array}{c}\text { Obesidade } \\
(n=43)\end{array}$} & \multirow[t]{2}{*}{ Valor $p$} \\
\hline & n & $(\%)$ & n & $(\%)$ & $\mathbf{n}$ & $(\%)$ & n & $(\%)$ & \\
\hline Insuficiente & 11 & $(47,9)$ & 29 & $(34,5)$ & 9 & $(24,4)$ & 16 & $(37,3)$ & 0,305 \\
\hline Adequado & 8 & $(34,8)$ & 29 & $(34,5)$ & 7 & $(18,9)$ & 10 & $(23,2)$ & 0,246 \\
\hline Excessivo & 4 & $(17,4)$ & 26 & $(31,08)$ & 21 & $(56,7)$ & 17 & $(39,5)$ & $0,009^{\star}$ \\
\hline
\end{tabular}

1 Institutes of Medicine; IOM (1990) 13.

* Baixo peso e eutrofia versus sobrepeso e obesidade.

demais grupos (Tabela 1). Quanto à idade gestacional em que receberam inicialmente a orientação dietética pela equipe de nutrição, verificou-se que o grupo de gestantes eutróficas iniciou mais tardiamente, com média de idade gestacional superior aos demais grupos.

A avaliação do consumo dietético de macronutrientes está demonstrada na Tabela 1 . Não se verifica diferença nas médias da quantidade diária de energia, nos diferentes grupos classificados pelo IMC pré-gestacional. Quanto ao perfil de macronutrientes, foi observada diferença significativa na média de ingestão de lípides: as gestantes de baixo peso apresentaram menor consumo de lípides quando comparadas ao grupo com eutrofia $(101,4$ versus $137,3 \mathrm{~g} ; \mathrm{p}=0,043)$. Quanto à proporção de distribuição dos macronutrientes, não foi verificada diferença significativa entre os grupos analisados.

O consumo dietético quanto à ingestão de micronutrientes está apresentado na Tabela 2. A ingestão diária de cálcio, vitamina $A$ e vitamina $C$ foi semelhante entre os grupos. Quanto à ingestão de ferro, a quantidade ingerida, por dia, foi maior nas gestantes eutróficas quando comparadas às com sobrepeso e com obesidade $(\mathrm{p}<0,001)$. A média diária de ingestão de folatos foi maior nas gestantes eutróficas quando comparadas às obesas $(\mathrm{p}=0,002) \mathrm{Na}$ avaliação da ingestão de fibras, observou-se que os resultados indicam menor ingestão nas gestantes baixo peso quando comparadas às eutróficas $(\mathrm{p}=0,042)$.

Quanto ao ganho ponderal materno, foi observada média significativamente menor $(\mathrm{p}<0,001)$ nas obesas (média $=8,4 \mathrm{~kg}, \mathrm{DP}=6,4 \mathrm{~kg}$ ) quando comparadas aos demais grupos: baixo peso (média $=12,5 \mathrm{~kg}, \mathrm{DP}=5,0$ $\mathrm{kg}$ ), eutrofia (média $=13,1 \mathrm{~kg}, \mathrm{DP}=5,2 \mathrm{~kg}$ ), e sobrepeso (média $=12,4 \mathrm{~kg}, \mathrm{DP}=5,8 \mathrm{~kg}$ ). $\mathrm{Na}$ análise em relação ao ganho ponderal total recomendado, verificou-se que o ganho ponderal materno total foi excessivo (acima do recomendado) em proporção significativamente maior $(\mathrm{p}=0,009)$ nas gestantes com sobrepeso e obesidade, quando comparadas às com baixo peso e eutrofia (Tabela 3 ).

\section{Discussão}

A avaliação do consumo dietético de gestantes, bem como o conhecimento do ganho ponderal materno, têm como objetivo trazer subsídios para o desenvolvimento de planos de ações eficazes no controle da qualidade da alimentação e no adequado ganho de peso, nesta fase da vida. A literatura demonstra que os extremos do estado nutricional antropométrico podem trazer riscos adicionais à gestação ${ }^{18-21}$. Conhecer os detalhes do consumo dietético aprimora a qualidade do atendimento e das orientações fornecidas. Aspectos das recomendações nutricionais podem ser essenciais para fortalecer o planejamento e desenvolvimento saudável da gravidez, minimizando riscos de comprometimento materno e perinatal.

O presente estudo verificou que a proporção de carboidratos no consumo dietético das gestantes variou de 55 a $59 \%$, conforme o estado nutricional antropométrico. Essa proporção deve sofrer influência dos hábitos alimentares 
de diferentes populações, pois em levantamento realizado na Inglaterra ${ }^{22}$, o consumo de carboidratos é de $223 \mathrm{~g} / \mathrm{d}$, que representa $50 \%$ do valor calórico total. Segundo estudo realizado no Rio de Janeiro ${ }^{23}$ o consumo médio de carboidratos é de $496 \mathrm{~g} / \mathrm{d}$, representando $65 \%$ do total energético, proporções estas diferentes das avaliadas neste estudo. O metabolismo dos carboidratos é importante determinante do crescimento fetal, fator este que pode contribuir para resultados perinatais adversos.

Quanto ao consumo proteico das gestantes, este estudo constatou média, em gramas, superior à observada em estudo de coorte prospectivo realizado na Inglaterra ${ }^{22}$, que foi de $67 \mathrm{~g} / \mathrm{dia}$. Além disso, a média de ingestão protéica nos grupos com eutrofia, sobrepeso e obesidade foi maior que o observado em estudo realizado na Nova Zelândia ${ }^{24}$, onde a média foi de $78 \mathrm{~g} / \mathrm{dia}$. Apesar de existirem diferenças socioeconômicas entre a população do presente estudo e as dos estudos internacionais citados, diferenças no método podem ter contribuído para as diferenças apontadas. Além disso, hábitos alimentares podem diferir com a diversidade cultural, o que pode influenciar nos resultados observados.

A ingestão de lípides, em todos os estados nutricionais antropométricos, demonstrou proporção média adequada quando comparada à ingestão energética total. Distribuição similar de lípides foi encontrada em estudo realizado no Japão ${ }^{25}$, onde a média de ingestão diária foi de $29 \%$ da recomendação energética total, porém Lof et al. $(2009)^{26}$ encontraram média de $82 \mathrm{~g}$, abaixo da quantidade encontrada no presente estudo. Apesar da proporção dos lípides em relação às calorias totais estarem dentro do padrão recomendado, sabe-se que, de maneira geral, a população brasileira tem como predominância de fonte lipídica as gorduras saturadas e trans, gorduras essas que são prejudiciais ao sistema cardiovascular. Nesse sentido, são necessários estudos específicos sobre o consumo de lípides na gestação, para que sejam conhecidos detalhes dos hábitos das gestantes do nosso meio, bem como desenvolvimento de estratégias para que a alimentação saudável possa ser estimulada.

Entre os micronutrientes analisados, o cálcio, o ferro, o folato e as fibras foram os que apresentaram menor ingestão em comparação às recomendações adotadas. A média encontrada de ingestão de cálcio esteve, em todos os estados nutricionais, abaixo da recomendada. As gestantes de baixo peso, seguidas pelas obesas, foram as que apresentaram ingestão média mais baixa, reforçando a necessidade de atenção nos extremos do estado nutricional. Freisling et al. (2006) ${ }^{27}$ verificaram ingestão diária de cálcio em gestantes e encontraram médias de 865 $\mathrm{mg} / \mathrm{d}, 950 \mathrm{mg} / \mathrm{d}$ e $1055 \mathrm{mg} / \mathrm{d}$, de acordo com o nível de escolaridade baixo, médio e alto, respectivamente. Estes valores são mais elevados que os observados na presente casuística, o que demonstra haver diferenças na qualidade da alimentação. Em estudo realizado em Londres ${ }^{28}$, é constatado consumo médio de cálcio de $883 \mathrm{mg} / \mathrm{d}$, também abaixo da recomendação, mas semelhante ao observado no presente estudo.

Quanto ao aporte de ferro no consumo dietético das gestantes do presente estudo, verificou-se que a média de ingestão diária está aquém das recomendações. Somente pela alimentação é difícil se atingir a quantidade recomendada, portanto, a partir do segundo trimestre, é imprescindível a suplementação vitamínica com compostos de ferro, além do incentivo à inclusão de alimentos fontes deste mineral, como carnes e vegetais de cor verde-escura.

A média de consumo de fibras dietéticas não atingiu a recomendação nos grupos analisados, sendo mais baixa nas gestantes de baixo peso. Este aspecto reflete, em parte, os hábitos de grande parte da população urbana brasileira com baixa ingestão de alimentos fonte de fibras, como frutas, legumes e verduras. Buss et al. $(2008)^{29}$, observaram, em gestantes, consumo médio de fibras de $30,2 \mathrm{~g} / \mathrm{d}$, porém $50,2 \%$ da amostra não atinge a recomendação.

Em estudo com grávidas de uma região urbana do Ira ${ }^{21}$, mulheres com baixo peso pré-gestacional demonstram risco para ganho ponderal insuficiente na gravidez, e, nas gestantes obesas, o ganho ponderal excessivo é observado em $47,9 \%$, proporção comparável ao presente estudo.

A limitação deste trabalho é o caráter retrospectivo, o que dificulta a análise do impacto da orientação nutricional oferecida como medida de intervenção. Além disso, o valor energético médio obtido nesta casuística é inferior ao observado na prática, o que pode significar certa impropriedade na análise dos questionários quanto a este parâmetro. Apesar de a entrevista ter sido realizada, em média, na metade da gravidez, acredita-se que não haja grande variação nos hábitos alimentares ao longo da gravidez. Na presente investigação, o ganho de peso excessivo, acima do recomendado, mais frequente nas gestantes com sobrepeso e obesidade, reflete o desafio em se obter sucesso na orientação dietética. Novas estratégias devem ser delineadas, bem como a captação precoce dessas mulheres para melhor orientação nutricional e conscientização da importância do ganho de peso na gravidez.

O conhecimento do consumo dietético da gestante brasileira é de grande relevância, bem como o seu estado nutricional pré-gestacional. A orientação dietética procura direcionar os hábitos alimentares para adequar a oferta de energia, macro e micronutrientes. No presente estudo, apesar da orientação nutricional oferecida, o excesso no ganho ponderal materno total associou-se ao sobrepeso e à obesidade, o que remete à reflexão sobre a real aderência dessas gestantes ao que é recomendado, bem como à forma 
com que essas orientações são transmitidas. O consumo dietético de gestantes acompanhadas em hospital universitário difere de acordo com o estado nutricional antropométrico pré-gestacional. As gestantes com sobrepeso e obesidade apresentam menor ingestão diária de ferro quando comparadas às eutróficas, e as obesas, menor ingestão de folatos. Isso reforça a importância da suplementação vitamínica pré-natal.

\section{Referências}

1. Jackson AA, Robinson SM. Dietary guidelines for pregnancy: a review of current evidence. Public Health Nutr. 2001;4(2B):625-30.

2. Kind KL, Moore VM, Davies M. Diet around conception and during pregnancy - effects on fetal and neonatal outcomes. Reprod Biomed Online. 2006;12(5):532-41.

3. Bang SW, Lee SS. The factors affecting pregnancy outcomes in the second trimester pregnant women. Nutr Res Pract. 2009;3(2): 134 40.

4. Picciano MF. Pregnancy and lactation: physiological adjustments, nutritional requirements and the role of dietary supplements. J Nutr. 2003;133(6): 1997S-2002S.

5. Zeisel SH. Is maternal diet supplementation beneficial? Optimal development of infant depends on mother's diet. Am J Clin Nutr. 2009;89(2):685S-7S

6. Rodrigues PL, Lacerda EMA, Schlüssel MM, Spyrides MHC, Kac G. Determinants of weight gain in pregnant women attending a public prenatal care facility in Rio de Janeiro, Brazil: a prospective study, 2005-2007. Cad Saúde Pública. 2008;24 Suppl 2:S272-S284.

7. Bertin RL, Parisenti J, Di Pietro PF, Vasconcelos FAG. Métodos de avaliação do consumo alimentar de gestantes: uma revisão. Rev Bras Saúde Matern Infant. 2006;6(4):383-90.

8. Siega-Riz AM, Viswanathan M, Moos MK, Deierlein A, Mumford $S$, Knaack J, et al. A systematic review of outcomes of maternal weight gain according to the Institute of Medicine recommendations: birthweight, fetal growth, and postpartum weight retention. Am J Obstet Gynecol. 2009;201(4):339.e1-14.

9. Rasmussen KM, Catalano PM, Yaktine AL. New guidelines for weight gain during pregnancy: what obstetrician/gynecologists should know. Curr Opin Obstet Gynecol. 2009;21 (6):521-6.

10. Konno SC, Benicio MHA, Barros AJD. Fatores associados à evolução ponderal de gestantes: uma análise multinível. Rev Saúde Pública. 2007;41 (6):995-1002.

11. Lagiou P, Tamimi RM, Mucci LA, Adami HO, Hsieh CC, Trichopoulos $D$. Diet during pregnancy in relation to maternal weight gain and birth size. Eur J Clin Nutr. 2004;58(2):231-7.

12. Hure A, Young A, Smith R, Collins $C$. Diet and pregnancy status in Australian women. Public Health Nutr. 2009;12(6):853-61.

13. Institute of Medicine. Subcommittee on Nutritional Status and Weight Gain During Pregnancy. Nutrition during pregnancy: part I, weight gain; part II, nutrient supplements. Washington, DC: National Academy Press; 1990.

14. Organização Mundial da Saúde (OMS). Dieta, nutrição e prevenção de doenças crônicas. Geneva: OMS; 2003. (Technical report series).

15. Institute of Medicine. Dietary reference intakes for vitamin $A$, vitamin $\mathrm{K}$, arsenic, boron, chromium, copper, iodine, iron, manganese, molybdenum, nickel, silicon, vanadium, and zinc. Washington, DC: National Academy Press; 2002.
16. Institute of Medicine. National Research Council. Dietary reference intakes for vitamin C, vitamin E, selenium, and carotenoids. Washington, DC: National Academy Press; 2002.

17. Institute of Medicine. Dietary reference intakes for energy, carbohydrate, fiber, fat, fatty acids, cholesterol, protein, and amino acids. Washington, DC: National Academy Press; 2005.

18. Kristensen J, Vestergaard M, Wisborg K, Kesmodel U, Secher NJ. Pre-pregnancy weight and the risk of stillbirth and neonatal death. BJOG. 2005; 1 12(4):403-8.

19. Padilha PC, Saunders C, Machado RCM, Silva CL, Bull A, Sally EOF, et al. Associação entre o estado nutricional pré-gestacional e a predição do risco de intercorrências gestacionais. Rev Bras Ginecol Obstet. 2007;29(10): 51 1-8.

20. Roman H, Robillard PY, Hulsey TC, Laffitte A, Kouteich K, Marpeau $L$, et al. Obstetrical and neonatal outcomes in obese women. West Indian Med J. 2007;56(5):42 1-6.

21. Yekta Z, Ayatollahi H, Porali R, Farzin A. The effect of pre-pregnancy body mass index and gestational weight gain on pregnancy outcomes in urban care settings in Urmia-Iran. BMC Pregnancy Childbirth. 2006;6:15.

22. Brion M, Ness AR, Rogers I, Emmett $P$, Cribb V, Davey Smith G, et al. Maternal macronutrient and energy intakes in pregnancy and offspring intake at $10 \mathrm{y}$ : exploring parental comparisons and prenatal effects. Am J Clin Nutr. 2010;91(3):748-56.

23. Lacerda EMA, Kac G, Cunha CB, Leal MC. Consumo alimentar na gestação e no pós-parto segundo cor da pele no município do Rio de Janeiro. Rev Saúde Pública. 2007;41 (6):985-94.

24. Watson PE, McDonald BW. Major influences on nutrient intake in pregnant New Zealand women. Matern Child Health J. 2009; 13(5):695-706.

25. Watanabe H, Fukuoka H, Sugiyama T, Nagai Y, Ogasawara $\mathrm{K}$, Yoshiike N. Dietary folate intake during pregnancy and birth weight in Japan. Eur J Nutr. 2008;47(6):341-7.

26. Lof M, Hilakivi-Clarke L, Sandin SS, de Assis S, Yu W, Weiderpass E. Dietary fat intake and gestational weight gain in relation to estradiol and progesterone plasma levels during pregnancy: a longitudinal study in Swedish women. BMC Womens Health. 2009;9:10

27. Freisling H, Elmadfa I, Gall I. The effect of socioeconomic status on dietary intake, physical activity and Body Mass Index in Austrian pregnant women. J Hum Nutr Diet. 2006;19(6):437-45.

28. Derbyshire E, Davies GJ, Costarelli V, Dettmar PW. Habitual micronutrient intake during and after pregnancy in Caucasian Londoners. Matern Child Nutr. 2009;5(1):1-9.

29. Buss C, Nunes MA, Camey S, Manzolli P, Soares RM, Drehmer M, et al. Dietary fibre intake of pregnant women attending general practices in southern Brazil - the ECCAGE Study. Public Health Nutr. 2009; $12(9): 1392-8$. 